\title{
Application of Surface Tension Measurement to Determined Saturated Cooking Oil and Unsaturated Cooking Oil
}

\author{
Adi Ahmad Dimisa*, Indira Prabawati Hanggara ${ }^{* *}$, Frida Agung Rakhmadi*** $^{* *}$ \\ Physics Department, Faculty of Science and Technology, UIN Sunan Kalijaga \\ Jl. Marsda Adisucipto No. 1 Yogyakarta 55281, Indonesia. Tel. + 62-274-540971, Fax. + 62-274-519739 \\ Email: adi.ahmad.dimisa@gmail.com*, Indihanggara@gmail.com ${ }^{* *}$, frida.rakhmadi@uin-suka.ac.id**
}

\begin{abstract}
Application surface tension measuring tool to distinguish saturated cooking oil and unsaturated cooking oil has been done. This study aims to apply the surface tension measuring tool as an alternative tool for distinguishing saturated cooking oil and unsaturated cooking oil. This study was conducted to distinguish the quality of cooking oil consumption in terms of the number of oil for frying. The method used in this research is the method Torsion Dynamometer conducted in three phases, the first preparation of tools and materials, the second data retrieval, and the third is data processing. The results showed that torsion Dynamometer can be used as a tool to distinguish saturated cooking oil and unsaturated cooking oil. Saturated cooking oil has a value of surface tension $\gamma \pm \Delta \gamma=(1.6604 \pm$ $0.01448)$ dyne $/ \mathrm{cm}$, while for unsaturated cooking oil has a value of surface tension $\gamma \pm \Delta \gamma=(1.3492 \pm 0.03624) \mathrm{dyne} / \mathrm{cm}$.
\end{abstract}

Keywords: Different test, Saturated cooking oil, Surface tension, Unsaturated cooking oil

\section{INTRODUCTION}

Cooking oil is a human need that is used as a food frying medium. Most humans consume fried foods (Rany, 2018). Frying is a process carried out by the community every day. In the frying process, cooking oil functions as a conductive heat medium, adding savory flavors and calories in food (Rany, 2018). In addition to calories, oil also contains fatty acids. The content of fatty acids in oil is divided into two conditions, namely saturated fatty acids and unsaturated fatty acids. Monounsaturated Fatty Acids (MUFA) is a type of fatty acid which has 1 (one) double bond in the carbon atom chain. While saturated fatty acids (SFA) are fatty acids that do not have double bonds on carbon atoms. This means saturated fatty acids are not sensitive to oxidation and free radical formation (Ratu Ayu, 2008).

Saturated fatty acids or commonly called saturated cooking oil can be produced from the frying process. Saturated cooking oil is that has been used for frying different kinds of food and have experienced a change in chemical composition (Rukmini, 2007). Damage to the oil is often referred to as rancidity. Damage to the oil during the frying process will affect the quality and nutritional value of food is fried (Thadeus, 2005).

The nutritional value contained in the foods fried in saturated oils are very dangerous if the body is too often consume. We also can not guarantee that the oils used by street vendors or in restaurant meal replaced the oil regularly. Therefore this research aims to determine the difference between saturated oils and unsaturated oils based on surface tension characteristics.

The surface tension is the surface properties of a liquid substance that behaves like a thin layer of skin that is supple as a result of the influence of tension (Alfahru, 2015). Mass density becomes the most important point in this research. Saturated oils tend to have a greater density than the unsaturated oils. This is what causes the surface tension of the saturated oils greater than unsaturated oils.

\section{MATERIALS AND METHODS}

\section{Tools and Materials}

Materials need to be prepared are two different oil samples. One sample of the unsaturated oils or has not been used for frying and a saturated oil sample that has been used 3 times a frying pan. The tools used in the retrieval of data is called Torsion Dynamometer.

\section{Data Collection Methods}

After preparation tools and materials have been done, the next step was to collect the data. The collecting of data using methods Torsion Dynamometer. Torsion Dynamometer method can be used to measure surface tension. The principle of this tool is the force required to detach a platinum-iridium ring immersed at the surface is equal to the surface tension of the liquid.

The data was collected by varying the measurement 5 times and the data collection process was in the laboratory room at room temperature $32^{\circ} \mathrm{C}$. The data have been obtained and analyzed how characteristics which can then be used to determine whether the data can be continued in the manufacture of detection system or not. If the data obtained intersect, then the data can not be used for the manufacture of detection system. If the data obtained do not intersect, it can be continued or be used for the manufacture of detection system. 


\section{Data Processing}

The above data processing method is by determining the surface tension using the equation,

$$
\gamma_{n}=\frac{F_{n}}{d}
$$

With $F_{n}$ is a force variation of the $n$ and $d$ is the diameter of the ring $(19 \mathrm{~mm})$. While the average value of the surface tension can be obtained by the equation,

$$
\bar{\gamma}=\frac{\sum \gamma_{n}}{n}
$$

Having obtained the magnitude of the surface tension on average, the next step is determining the error of the experiment by using the equation,

$$
\Delta \gamma_{n}=\left|\gamma_{n}-\bar{\gamma}\right|
$$

With $\Delta \gamma_{n}$ is error of the surface tension n-data. Then the average value of surface tension error can be obtained by the equation,

$$
\overline{\Delta \gamma}=\frac{\sum \Delta \gamma_{n}}{n}
$$

\section{RESULTS AND DISCUSSION}

\section{Data Processing}

The results of data processing by equation 1 are shown in Table 1.

Table 1. Results of data processing by equation 1 .

\begin{tabular}{ll}
\hline $\begin{array}{l}\text { Saturated Cooking Oils } \\
\text { (dyne/cm) }\end{array}$ & $\begin{array}{l}\text { Unsaturated Cooking Oils } \\
\text { (dyne/cm) }\end{array}$ \\
\hline 1.684 & 1,421 \\
1,657 & 1,321 \\
1,631 & 1.368 \\
1,657 & 1,315 \\
1,673 & 1,321 \\
\hline
\end{tabular}

The average value obtained from the results of data processing using equation 2 was 1.3492 dyne/cm for a sample of unsaturated oils and $1.6604 \mathrm{dyne} / \mathrm{cm}$ for samples saturated oils.

Then the average error by using equation 4 was obtained surface tension value of saturated cooking oils is \pm 0.01448 dyne $/ \mathrm{cm}$, while the unsaturated cooking oil is $\pm 0.03624 \mathrm{dyne} / \mathrm{cm}$.

Thus the data processing of saturated oil surface tension $\gamma \pm \Delta \gamma=(1.6604 \pm 0.01448)$ dyne $/ \mathrm{cm}$, while the unsaturated cooking oil has a surface tension $\gamma \pm \Delta \gamma=$ $(1.3492 \pm 0.03624)$ dyne $/ \mathrm{cm}$.

Analysis of the data from this research was obtained, in the case of saturated oils, mass density that play a role in the magnitude of the surface tension. Saturated oil which is a result of the process of frying oil several times will have a greater density. Based on the results of the processing of the data contained in table 1.1 is proved that the surface tension of saturated cooking oil greater than unsaturated cooking oil. This is consistent with the theory that the magnitude of the surface tension is influenced by several factors, such as the type of fluid, temperature, pressure, mass density, solute concentration, and mass density.

From the data processing show that samples of saturated cooking oil and unsaturated oil do not intersect, where the sample is saturated cooking oil are in the range of 1.6 whereas the range of unsaturated oil samples was in the range of 1.3 range. Such data can be clearly distinguished from the value of surface tension. Therefore, from these data can be used as a basic data for the manufacture of detection system.

\section{CONCLUSIONS}

Base on research done, it can be conclude that torsion dynamometer can be used as a tool to distinguish saturated cooking oil and unsaturated cooking oil. Saturated cooking oil has a value of surface tension $\gamma \pm$ $\Delta \gamma=(1.6604 \pm 0.01448)$ dyne/cm, while for unsaturated cooking oil has a value of surface tension $\gamma$ $\pm \Delta \gamma=(1.3492 \pm 0.03624)$ dyne/cm. Therefore, from these data can be used as a basic data for the manufacture of detection system.

\section{ACKNOWLEDGMENTS}

Thanks authors say to all those who have supported and helped research as well as in writing. We say thanks to Mr. Frida Agung Rakhmadi the our supervisor who gave ideas and guidance. We would like to thank the head of the basic physics lab, Mr. Ashim who was willing to help procure torsion dynamometer.

\section{REFERENCES}

Mangidi, Alfahru. 2015.Practice report Physical Chemistry II Experiment II Determination of Surface Tension Creep Capillary Fluid Method. Haluoleo university Kendari.

Maryani, H., Rukmini. And Ward, YF. (2007) "Participation of communities, Cross Private Sector In Posyandu revitalization". Health Systems Research Bulletin, Vol. 10, No. April 2nd; 145150.

Thandeus MS. 2005. Effect of Vitamin C and Vitamin E Against Liver histological changes, heart and aorta Musmusculus L Gulur Switzerland derrived Aubat Provision Used Cooking Oil. [Thesis]. University of Indonesia.

Tuasamu,Rany.2018.https://www.researchgate.net/publication/3252 02881_Bahaya_Penggunaan_Minyak_PengGorengan_Berulang _Dilihat_Dari_Angka_Peroksidanya (Accessed on 8 October 2018). 